\title{
ESTUDO DE ASSOCIAÇÃO \\ ENTRE FORÇA MUSCULAR E \\ MASSA MAGRA EM MULHERES IDOSAS
}

\section{DR. RICARDO MORENO LIMA}

Doutorado em Educação Física na Universidade Católica de Brasília (UCB). Professor Adjunto da Faculdade de Educação Física, Universidade de Brasília (UnB)

(Brasília -Distrito Federal - Brasil)

E-mail: ricardomoreno@unb.br

\section{MS. CARLOS ERNESTO SANTOS FERREIRA}

Mestrado em Educação Física na Universidade Católica de Brasília (UCB).

Professor Adjunto do Curso de Educação Física, Universidade Católica de Brasília (UCB)

(Brasília -Distrito Federal - Brasil)

E-mail:ernestobsb@gmail.com

\section{DRA. LIDIA MARA AGUIAR BEZERRA}

Doutorado em Educação Física na Universidade Católica de Brasília (UCB).

Professora adjunta do Centro Universitário Euro-Americano (UNIEURO)

(Brasília -Distrito Federal - Brasil)

E-mail: lidia.bezerra@gmail.com

\section{DRA. HELOISA THOMAZ RABELO}

Doutorado em Educação Física na Universidade Católica de Brasília (UCB) Professora adjunta no

Centro Universitário do Leste de Minas Gerais (UNILESTEMG)

(Coronel Fabriciano - Minas Gerais - Brasil)

E-mail: htrrrs@terra.com.br

\section{PROF. LUIZ CARLOS DA SILVA JÚNIOR}

Mestrando em Educação Física na Universidade Católica de Brasília (UCB).

Professor Adjunto do curso de Educação Física, Universidade Estadual Vale do Acaraú (UVA).

(Sobral - Ceará - Brasil)

E-mail: Icjpersonal@hotmail.com

\section{MS. ENIVALDO PEREIRA DOS SANTOS}

Mestrado em Educação Física na Universidade Católica de Brasília (UCB).

Professor do Instituto Federal do Piauí (IFPI) (Teresina - Piauí - Brasil)

E-mail: enivaldopereira@bol.com.br

\section{DR. RICARDO JACÓ DE OLIVEIRA}

Doutorado em Neurologia pela Universidade Federal de São Paulo (UNIFESP).

Professor adjunto da Faculdade de Educação Física, Universidade de Brasília (UnB)

(Brasília -Distrito Federal - Brasil)

E-mail: rjaco@unb.br 


\section{RESUMO}

O objetivo do presente estudo foi verificar a associação entre a força muscular isocinética da musculatura extensora do joelho do membro dominante com a MM de diferentes segmentos

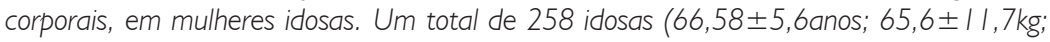
I,53 $\pm 0,07 \mathrm{~m}$ ) foram submetidas à avaliação isocinética do pico de torque (PT) e da MM (DXA). Correlações de Pearson foram examinadas entre os índices de MM dos braços, das pernas, do tronco, do corpo inteiro, da perna dominante (MMB, MMP, MMT, MMI, MMPD, respectivamente) com o PT. O nível de significância foi de $\leq 0,05$. Após análise dos dados, foi observado que a MM apresentou correlação positiva e significante com o PT. A correlação foi mais consistente à medida que a MM avaliada era mais próxima a musculatura da avaliação (MMPD), (MMT=0,3l, MMB =0,39, MMl=0,52, MMP=0,55, MMPD = $0,60)$. Com base nos resultados observados, concluímos que a MM é um dos importantes determinantes do PT em idosas.

PALAVRAS-CHAVE: Idosas; sarcopenia; massa magra; força muscular.

\section{INTRODUÇÃO}

Um fenômeno mundial chamado envelhecimento populacional coloca o Brasil como o país que terá a sexta população do mundo em número absoluto de idosos em 2030 (IBGE, 2009). As doenças advindas do envelhecimento geram elevados custos assistenciais em saúde. De acordo com a avaliação do centro nacional de estatística para a saúde, aproximadamente 84\% das pessoas com 65 anos ou mais são dependentes diretos para realizarem as atividades da vida diária, constituindo-se na maior ameaça de institucionalização. Ainda de acordo com os dados do IBGE (2009), em 2020, estima-se que ocorrerá aumento de 84 a 167\% de idosos com moderada ou grave incapacidade. Contudo, com a introdução de medidas preventivas nas quais se insere a prática de atividade física regular e programas de reabilitação, é possível melhorar a função fisiológica e minimizar ou prevenir o aparecimento de incapacidade. (SCHUARTZ, EVANS, 1995).

Segundo Raso et al. ( 1997), o envelhecimento é caracterizado pela redução gradativa da capacidade dos vários sistemas orgânicos. Dentre esses sistemas, pode-se destacar o muscular, o qual está envolvido em funções como sustentação, locomoção e respiração. A dependência funcional diretamente associada à perda da força muscular é a que mais aparece pelas alterações provocadas com o avanço da idade. (RASO et al., 1997). O termo sarcopenia surgiu no início da década de 90, referindo-se à redução da força e massa magra (MM) relacionada à idade. (ROSEMBERG, 1989; SCHUARTZ, EVANS 1995). Atualmente, esta denominação refere-se amplamente à perda gradual de massa muscular esquelética, ao passo que a redução da força e fatores associados, como propriedades contráteis e funções neurológicas, vêm também sendo definidos como dinapenia. (CLARK; MANINI, 2008). 
Estudos têm evidenciado a associação entre Sarcopenia e inúmeros problemas de saúde tais como perda da independência funcional, maior número de quedas, osteoporose, disfunção da termorregulação e intolerância à glicose. (SILVA et al. 2006; LIMA et al., 2009; OLIVEIRA et al., 2009). De fato, relatos prévios fornecem evidências de que a sarcopenia apresenta relevante implicação nos custos assistenciais em saúde (JANSSEN et al., 2004). Sendo assim, fica nítida a necessidade de estudos que determinem os fatores que levam à perda da força muscular associada ao envelhecimento.

Segundo Lindle (1997), força e massa muscular apresentam estreita relação, portanto, a sarcopenia caracteriza-se também por uma concomitante redução de força. Entretanto, outros fatores também são apontados em determinar a perda de força muscular decorrente do envelhecimento. Com o avançar da idade ocorrem alterações na estrutura e composição muscular, com perda de proteína contrátil e aumento de tecido conjuntivo e gorduroso (RICE, 1989), conferindo uma menor qualidade contrátil e reduzida produção de força para um mesmo volume ao músculo senescente. Outro importante fator que contribui para o declínio da força muscular está relacionado a aspectos neuromusculares, envolvendo a perda de motoneurônios, unidades responsáveis pela inervação das fibras musculares, e redução de sua taxa de inervação. (DOHERTY et al., 1993). Embora estudos prévios tenham determinado a relação entre massa e força muscular, poucos se preocuparam em examinar essa relação de forma mais específica, por exemplo, verificando a relação entre a massa muscular de um determinado membro e a força gerada por esse mesmo segmento corporal. (CAULEY et al., 1987).

A avaliação isocinética tem sido sugerida e frequentemente utilizada tanto na rotina clínica como em protocolos de pesquisa. (DROUIN et al., 2004). Adicionalmente, o dinamômetro isocinético vem sendo amplamente utilizado tanto para caracterizar e avaliar indivíduos idosos (BOTTARO et al., 2005) quanto no treinamento propriamente dito (ERNESTO et al., 2009). Neste sentido, a determinação dos fatores que levam à perda de força comum ao processo de envelhecimento auxiliará no entendimento da sarcopenia e fornecerá informações importantes para o delineamento de programas preventivos e terapêuticos.

Dessa forma, o objetivo do presente estudo foi verificar a associação entre a força muscular isocinética da musculatura extensora do joelho do membro dominante com a MM de diferentes segmentos corporais, em muIheres idosas. 


\section{AMOSTRA}

A amostra do presente estudo foi constituída por 258 idosas, com idade compreendida entre 57 a 86 anos, praticantes de atividades físicas regulares, participantes de um projeto social desenvolvido na Universidade, e selecionadas por conveniência. Após avaliação médica e aplicação dos critérios de exclusão, todas as voluntárias foram submetidas à avaliação da composição corporal e do Pico de Torque (PT) da musculatura extensora do joelho. Foram adotados os seguintes critérios de exclusão: mulheres que não tinham nacionalidade brasileira; caminhar somente com assistência; possuir qualquer tipo de prótese de quadril ou outra articulação que comprometesse a execução do movimento de avaliação isocinética; tabagismo; e desordem metabólica ou endócrina que sabidamente afetasse o sistema muscular.

Antes do início da coleta de dados, foi dada entrada do projeto junto ao comitê de ética em pesquisa da Universidade Católica de Brasília para a devida apreciação, sendo o protocolo utilizado na metodologia aprovado sob o oficio CEP/ UCB n ${ }^{\circ}$ 072/2004. Todas as participantes assinaram um termo de consentimento livre e esclarecido, o qual destacava os possíveis riscos e benefícios referentes à participação na pesquisa.

\section{AVALIAÇÃO MÉDICA INICIAL}

As voluntárias foram submetidas à avaliação cardiológica no Laboratório de Estudos em Educação Física e Saúde (LEEFS/UCB), composta de Eletrocardiograma de repouso (ECG), por meio de eletrocardiógrafo digital (Elite Micromed $®)$, medição da pressão arterial (PA) e anamnese específica (ANM) por um médico cardiologista, o qual acompanhou cada voluntária durante a execução dos demais testes. Caso fosse diagnosticado pelo Cardiologista, em qualquer voluntária, algum problema durante os testes cardiológicos (ECG, PA, ANM) que pudesse comprometer tanto a saúde da mesma quanto à execução dos testes, a voluntária era excluída da amostra.

\section{AVALIAÇÃO DA COMPOSIÇÃO CORPORAL}

As voluntárias foram avaliadas por meio de uma balança digital (TOLEDO Beyond Technology, modelo PL - 200® - São Paulo - Brasil) com escala de 0,05 Kg.

Para a variável estatura foi utilizado o estadiômetro (COUNTRY TECNOLOGY INC ${ }$, Gays Mills, WI) com escala de 0,1 cm. As mensurações de composição corporal foram realizadas no laboratório de imagens da Universidade por meio 
de absortometria por raios- $x$ de dupla energia (DXA), e para tanto foi utilizado o equipamento da marca Lunnar, modelo DPX-IQ (Lunar Corporation ${ }^{\circledR}$, Madison, WI, USA). O referido método foi utilizado por ser considerado válido para avaliar a composição corporal de mulheres idosas, podendo ser aplicado para mensurar a MM dessa população. (HANSEN et al. 1999).

Para o procedimento, as voluntárias se posicionaram em decúbito dorsal sobre a mesa do equipamento, sendo em seguida cuidadosamente dispostas de forma que ficassem totalmente centralizadas em relação às laterais da mesa. Após análise de toda a área corporal, o DXA possibilitou a determinação da densidade mineral óssea e dos tecidos. Os tecidos foram ainda fracionados em Massa de gordura e MM. Os apêndices (membros superiores e inferiores) foram isolados do tronco e da cabeça utilizando linhas geradas pelo programa, as quais, em seguida, foram manualmente ajustadas por um técnico devidamente treinado para esse fim. Dessa forma, além do equipamento fornecer valores de MM e massa de gordura do corpo inteiro, foi possível também identificar valores para as seguintes regiões corporais: membros superiores, inferiores e tronco, sendo ainda possível distinguir membro direito do membro esquerdo.

\section{AVALIAÇÃO DA FORÇA MUSCULAR DO MEMBRO INFERIOR DOMINANTE}

A força do quadríceps foi mensurada utilizando-se o dinamômetro isocinético Biodex System 3 (Biodex Medical Systems ${ }^{\circledR}$, New York, USA) no Laboratório de Avaliação Física e Treinamento (LAFIT/UCB). Antes do teste, as participantes foram submetidas a um aquecimento em cicloergômetro utilizando cargas leves. Após explicação detalhada dos procedimentos da avaliação, as voluntárias foram cuidadosamente posicionadas no assento do equipamento. $\bigcirc$ eixo de rotação do braço do dinamômetro foi alinhado com o epicôndilo lateral do fêmur dominante das voluntárias. O posicionamento do acessório foi aplicado aproximadamente a dois centímetros do maléolo medial. Cintos fixadores foram utilizados no tronco, pelve e coxa no intuito de minimizar eventuais movimentos compensatórios e possível auxílio de outros grupamentos musculares na produção do torque não específicos a musculatura extensora do joelho.

Após familiarização com o equipamento, foi realizado o teste propriamente dito, utilizando protocolo constituído de três séries de quatro contrações musculares na velocidade angular de $60 \%$ s. Foi aplicado intervalo de recuperação de 30 segundos entre as séries. (BOTTARO; OLIVEIRA, 2005). O valor registrado para análises posteriores foi o maior pico de torque (PT) das três séries, o qual foi expresso em valores absolutos $\left(\mathrm{N} \bullet \mathrm{m}^{-1}\right)$ e relativos à massa corporal $\left(\mathrm{N} \bullet \mathrm{m}^{-1} / \mathrm{kg}\right)$. 
Às participantes foi solicitado que realizassem as contrações, com o maior vigor possível. Encorajamento verbal e feedback visual foram fornecidos às voluntárias. A calibração do equipamento foi realizada de acordo com as instruções do fabricante no início das sessões de avaliação. Todos os testes foram realizados pelo mesmo avaliador treinado no procedimento.

\section{TRATAMENTO ESTATÍSTICO}

Os dados foram apresentados por meio da estatística descritiva, utilizando-se os procedimentos de média e desvio padrão. Para verificar a relação entre força muscular e a MM das diferentes regiões corporais foram conduzidos testes de correlação de Pearson. $\bigcirc$ nível de significância adotado foi valor de $p \leq 0,05$. $\bigcirc$ software utilizado para realização das análises citadas foi o SPSS para Windows versão I 5.0 com licença de uso.

\section{RESULTADOS}

As características descritivas da amostra estudada são apresentadas na Tabela I .

Tabela I. Características descritivas das participantes. Os valores são expressos como média \pm Desvio Padrão

\begin{tabular}{lc}
\hline Variáveis & \\
\hline $\mathrm{N}$ & 258 \\
Idade (anos) & $66,8 \pm 5,6$ \\
Massa Corporal (Kg) & $65,6 \pm 11,7$ \\
Estatura (m) & $1,53 \pm 0,07$ \\
IMC $\left(\mathrm{Kg} / \mathrm{m}^{2}\right)$ & $28,03 \pm 4,49$ \\
PT absoluto $\left(\mathrm{N} \cdot \mathrm{m}^{-1}\right)$ & $94,31 \pm 22,51$ \\
MMB (g) & $3198,5 \pm 869,7$ \\
MMP (g) & $11344,7 \pm 1887,3$ \\
MMTronco (g) & $21047,4 \pm 5072,2$ \\
MMInteiro (kg) & $37,7 \pm 5,2$ \\
MMPD (g) & $5644,2 \pm 819,0$ \\
\hline IMC = Índice de Massa Corporal; PT = Pico de Torque Isocinético; MMB = Massa Magra dos Braços; MMP = \\
Massa Magra das Pernas; MMT = Massa Magra do Tronco; MMI = Massa Magra Inteiro; MMPD = Massa Magra \\
Perna Direita:
\end{tabular}

A Tabela 2 apresenta as correlações entre o PT isocinético, idade das voluntárias e as variáveis de MM. De forma esperada, a idade das voluntárias se correlacionou negativa e significativamente com a força muscular, sugerindo que, 
mesmo após os sessenta anos, a força muscular declina com o avançar da idade. Todas as variáveis de MM relacionaram-se positiva e significativamente com a força muscular. De forma interessante, a correlação entre as variáveis foi maior à medida que a MM avaliada era aquela mais próxima ao local da avaliação de força, isto é, o membro inferior dominante. Por exemplo, uma correlação de 0,3 I foi observada entre MM do tronco e força, enquanto que uma correlação de 0,60 foi encontrada com a MM do membro dominante.

Tabela 2. Valores de correlação entre o pico de torque isocinético e variáveis da massa magra e idade.

\begin{tabular}{c|c|c|c|c|c|c}
\hline & Idade & MMB & MMP & MMT & MMI & MMPD \\
\hline $\mathrm{PT}\left(\mathrm{N} \cdot \mathrm{m}^{-1}\right)$ & $-0,31 *$ & $0,39 *$ & $0,55 *$ & $0,31 *$ & $0,52 *$ & $0,60 *$ \\
\hline
\end{tabular}

MMB = Massa Magra dos Braços; MMP = Massa Magra das Pernas; MMT = Massa Magra do Tronco; $\mathrm{MMI}=$ Massa Magra Inteiro; MMPD = Massa Magra Perna Direita. * Correlação significativa entre as variáveis $(P<0,00 \mathrm{I})$

\section{DISCUSSÃO}

Os achados do presente estudo demonstraram que a força muscular se correlaciona de forma mais expressiva entre a MM da região avaliada em comparação com a MM que não é da região avaliada. Essas observações sugerem que o volume muscular expresso por meio da MM é uma variável importante para a determinação da força muscular isocinética em mulheres idosas. Visto que a sarcopenia relaciona-se com consequências negativas à saúde do idoso, é de suma importância que essa população seja submetida a intervenções que minimizem a perda de força e massa muscular comumente observada com o processo de envelhecimento.

Segundo Kostka (2005), o fato de se utilizar a musculatura do quadríceps em testes isocinéticos se justifica em virtude de a mesma ser excelente preditora de incapacidade física em indivíduos idosos. Estudos prévios fornecem evidência de que a força dos extensores do joelho, quando preservada, está associada a um melhor desempenho nas atividades da vida diária e até mesmo maior prevenção de quedas. (ORDWAY et al. 2006; GURALINK et al. 1995). Estudos têm demonstrado o efeito do envelhecimento na força muscular, principalmente da musculatura extensora do joelho em idosos. Estudo longitudinal com duração de 07 anos demonstrou redução de 3,2\% da força por ano em 23 homens com idade entre 73 a 86 anos. (ANIANSSON et al. 1986). Com relação às contrações isocinéticas, Frontera et al. (2000) observaram redução de 30\% do pico de torque (PT), assim como também foi observada significante redução na área de secção transversa. Embora resultados prévios e os do presente estudo demonstrem a importância do volume muscular em determinar a força de mulheres idosas, outros fatores precisam ser considerados. Sendo assim, as moderadas correlações observadas no presente estudo, embora 
significativas, apontam que outros fatores contribuem para o declínio de força com o envelhecimento. Por exemplo, a perda de proteína contrátil e o aumento de tecido conjuntivo e gorduroso na estrutura do músculo (RICE, 1989), conferindo uma menor qualidade contrátil e reduzida produção de força para um mesmo tamanho de músculo. Outro importante fator que contribui para o declínio da força muscular é a perda de motoneurônios. (DOHERTY; VANDERVOORT; LEXELL, 1993). Estudos que utilizaram eletromiografia (STALBERG, 1980) ou técnicas para estimar o número de unidades motoras (DOHERTY et al., 1995) demonstraram perdas substanciais das unidades motoras ativas em músculos das extremidades superiores e inferiores. (DEKONING et al., 1988; DOHERTY; BROWN, 2002). Esse processo é apontado como outro importante fator em explicar a perda de força muscular característica do avançar da idade.

Segundo Izquierdo et al. (200 I), a redução da massa muscular é resultado das alterações hormonais, apesar de Malkia et al. (1994) associarem também a redução da força muscular à diminuição da quantidade e qualidade da atividade física. Estudos como o de Kostka et al. (2000) evidenciam que com o envelhecimento é notória a redução dos níveis de hormônios anabólicos, como o de crescimento insulínico I (IGFI) e testosterona. Fleck e Kraemer ( 1997) relatam que ocorre um decréscimo nos valores de repouso dos hormônios anabólicos como testosterona, hormônio do crescimento e insulina, bem como a resposta destes ao treinamento de força. Em estudo comparando idosos e jovens, Urso et al. (2006) observaram perda da massa muscular do adutor do polegar significativa somente em idosos quando submetidos à imobilização dessa musculatura, sendo que no mesmo estudo os autores puderam constatar redução da força muscular de forma semelhante nos dois grupos, atribuindo desta forma a compensação da atrofia aos fatores neurais. Somado a isso, o processo de perda de força tem sido atribuído em grande parte ao declínio de MM característico do envelhecimento. (FRONTERA et al. 2000). Os achados observados no presente estudo corroboram essas evidências, uma vez que a correlação negativa entre idade e massa magra indica que o envelhecimento está associado ao declínio de volume muscular. Ademais, esse declínio de volume muscular determina, em parte, a concomitante redução de força.

Visto que a perda de MM e força muscular repercutem negativamente sobre a saúde e independência dos idosos, estratégias que minimizem esses declínios contribuirão para qualidade de vida dessa população. A inatividade física é um fator importante em contribuir para o desenvolvimento da sarcopenia. De fato, é bem constatado na literatura que indivíduos idosos menos ativos apresentam menor massa muscular e maior incapacidade em realizar atividades da vida diária, quando comparados a seus congêneres mais ativos. (PORTER; VANDERWOORT, I995; EVANS, 2002). 
Várias formas de intervenção têm sido propostas para a prevenção e tratamento da sarcopenia, sendo o treinamento resistido um tipo de atividade que vem recebendo grande atenção nesse sentido. Segundo o Colégio Americano de Medicina do Esporte (ACSM, 2002), a força muscular é uma variável da aptidão física imprescindível para a manutenção da saúde, bem como da habilidade funcional e da qualidade de vida. $\bigcirc$ treinamento resistido sistematizado resulta em adaptações morfológicas, como aumento da massa muscular, aumento nos níveis de força, bem como redução da gordura corporal. (IZQUIERDO et al. 2004; TRAPPE; WILLIAMSON; GODARD, 2002; HUNTER; TREUTH, 1995). Entretanto, a inclusão deste tipo de treinamento para a população em questão tem sido recomendada somente nas duas últimas décadas.

Nesse sentido, resultados de inúmeros estudos demonstraram que os exercícios resistidos podem amenizar e até reverter um quadro de sarcopenia, provendo evidências de que esse tipo de treinamento deve ser contemplado dentro de um programa global voltado para pessoas idosas. (FIATARONE et al., 1990; PORTER; VANDERWOORT; LEXELL, 1995; EVANS, 2002). Por exemplo, Frontera et al. ( 1 988) observaram aumento de força da musculatura extensora do joelho em I07\% e de 227\% na musculatura flexora da referida articulação após serem submetidos a um programa de treinamento para obtenção da força, constituído de 8 repetições por série, sendo 3 séries por dia, com frequência semanal de 3 vezes, e a carga mantida a $80 \%$ da repetição máxima (IRM) durante 12 semanas. Os ganhos de força citados foram associados à significativa hipertrofia muscular e à taxa de renovação das proteínas musculares. Sendo assim, a literatura fornece evidências de que o treinamento de força é uma intervenção eficaz na prevenção e tratamento de sarcopenia. (ROTH; FERRELL; HURLEY, 2000).

Com base nos resultados observados, conclui-se que há associação entre a MM e a força muscular isocinética do membro inferior dominante. Adicionalmente, essa associação torna-se mais forte à medida que a MM analisada é aquela mais próxima do grupo muscular avaliado para força muscular isocinética. Em geral, esses resultados sugerem que a MM é, dentre outros, um importante determinante da força muscular de mulheres idosas. Esses achados contribuem para um melhor entendimento do fenômeno da sarcopenia, auxiliando ações dos profissionais de saúde na elaboração de intervenções.

\footnotetext{
Association between muscle strength and fat-free mass in older women ABSTRACT: The purpose of the present study was to correlate knee extensors muscle strength with fat-free mass (FFM) of different body sites in older women. Peak torque (PT) from 258
} 
women (66.58 $\pm 5.6 y e a r s ; 65.6 \pm 11.7 \mathrm{~kg} ; 1.53 \pm 0.07 \mathrm{~m})$ was assessed using an isokinetic dynamometer (Biodex) and FFM was evaluated using dual-energy x-ray absorptiometry (DXA). Pearson correlations were performed between FFM from Arms, Legs, Thorax, Whole Body and Dominant Leg (FFMA, FFMLs, FFMT, FFMWB and FFMDL, respectively) with PT. Significance level was set at $\square \leq 0.05$ for all comparisons. Positive and significant correlations were observed between FFM from all sites and isokinetic PT $(p<0.001)$. However, it was stronger when the site of FFM analyzed was closer to the muscle responsible to perform knee extension (FFMT $=0.31 ; F F M A=0.39 ; F F M W B=0.52 ; F F M L s=0.55 ; F F M D L=$ 0.60). Based on the observed results, it can be concluded that FFM is one of the important determinants of isokinetic muscle strength in older women.

KEYWORDS: Older; sarcopenia; fat-free mass; strength.

\section{Estudio de la asociación entre fuerza muscular y masa magra en mujeres mayores}

RESUMEN: El objetivo de este estudio fue correlacionar la fuerza muscular de los extensores de la rodilla con la Masa Magra (MM) en diferentes segmentos corporales en mujeres mayores.

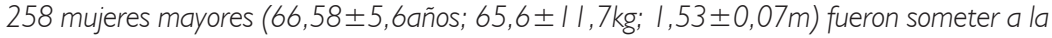
evaluación del pico de torque (PT) en el dinamómetro (Biodex) y de la MM (DXA). Correlaciones de Pearson se realizó entre la MM de los brazos, piernas, tronco, el cuerpo entero, de la pierna dominante (MMB, MMPs, MMT, MMCl, MMPD, respectivamente) con el PT. La MM de todas regiones examinadas presentaron correlación positiva y significante con los valores del PT $(p<0,001$ ), entretanto las correlaciones fueron más fuertes en la medida que la MM calculada era más próxima a musculatura utilizada en la evaluación del PT (MMPD), $(M M T=0,31, M M B=0,39, M M C l=0,52, M M P s=0,55, M M P D=0,60)$. En conclusión, la $M M$ es una importante variable y posiblemente determinante para la fuerza muscular en las mujeres mayores.

PALABRAS CLAVE: Mayores; sarcopenia; masa magra; fuerza muscular.

\section{REFERÊNCIAS}

AMERICAN COLLEGE OF SPORTS MEDICINE (ACSM). Progression models in resistance training for healthy adults. Medicine and Science in Sports and Exercise, Hagertown, v. 34, n. 2, p. 364-380, feb. 2002.

ANIANNSON, A. et al. Muscle morphology, enzymatic activity, and muscle strength in elderly men: a follow up study. Muscle Nerve, v. 9, n. 7, p. 585-591, sept. 1986.

BOTTARO M.; RUSSO A. F.; OLIVEIRA, R. J. The effects of rest interval on quadriceps torque during an isokinetic testing protocol in elderly. Journal of Sports Science and Medicine, v. 4, p. 285-290, sept. 2005.

CAULEY J. A, et al. The decline of grip strength in the menopause: relationship to physical Nactivity, estrogen use, and anthropometric factors. Journal of Chronic Disease, v. 40, n. 2, p. I15-120, jan. 1987. 
CLARK, B. C.; MANINI, T. M. Sarcopenia $\neq$ dynapenia. The Journals of Gerontology Series A: Biological Sciences and Medical Sciences, v. 63, n. 8, p. 829-834, aug. 2008.

DEKONING, P. et al. Estimation of the number of motor units based on macro-EMG. Journal of Neurology Neurosurgery \& Psychiatry, London, v. 5I , p. 403-4I I, mar. 1988.

DOHERTY, T. J.; VANDERVOORT, A. A.; BROWN, W. F. Effects of ageing on the motor unit: a brief review. Canadian Journal of Applied Physiology, Champaign, v. I8, n. 4, p. 331358, dec. 1993.

DOHERTY, T. et al. Methods for estimating the numbers of motor units in human muscles. Journal of Clinical Neurophysiology, New York, v. I2, n. 6, p. 565-584, nov. 1995.

DOHERTY, T. J.; BROWN, W. F. Motor unit number estimation: methods and aplications. In: BROWN, W.F;; BOLTON, C. F. Neuromuscular function and disease: basic, clinical, and electrodiagnostic aspects. Philadelphia: Saunders, 2002. p. 274-290.

DROUIN, J. M. et al. Reliability and validity of the Biodex System 3 pro isokinetic dynamometer velocity, torque and position measurements. European Journal of Applied Physiology, Heidelberg, v. 91 , n. I, p. 22-29, jan. 2004.

ERNESTO, C., et al. Effects of different rest interval on isokinetic muscle performance among older adults. Revista Brasileira de Fisioterapia, São Carlos, v. 13, p. 65-72, feb. 2009.

EVANS, W. J. Effects of exercise on senescent muscle. Clinical Orthopaedics and Related Research, Philadelphia, v. 403 Suppl, p. S2II-S220, oct. 2002.

FIATARONE, M. A. et al. High-Intensity strength training in nonagenarians: effects on skeletal muscle. JAMA, Chicago, v. 263, n. 22, p. 3029-3034, jun. 1990.

FLECK, S. J.; KRAEMER, W. J. Designing resistance training programs, $2^{\text {nd }}$ ed. Champaign: Human Kinetics, 1997. p. I- I I5.

FRONTERA, W. R. et al. Aging skeletal muscle: a I2-yr longitudinal study. Journal of Applied Physiology, Bethesda, v. 88, p. 1321-1326, apr. 2000.

FRONTERA, W. R. et al. Strength conditioning in older men: skeletal muscle hypertrophy and improving function. Journal of Applied Physiology, Bethesda, v. 64, p. 1038-1044, mar. 1988.

GURALNIK, J.; M. et al. Lower-extremity function in persons over the age of 70 years as a predictor of subsequent disability. New England Journal of Medicine, Waltham, v. 332, n. 9, p. 556-56I, mar. 1995.

HANSEN R. D. et al. Determination of skeletal muscle and fat-free mass by nuclear and dual-energy $\mathrm{X}$-ray absorptiometry methodsin men and women aged 5I-84 y. American Journal of Clinical Nutrition, New York, v. 70, p. 228-233, aug. 1999. 
HUNTER, G. R.; TREUTH, M. S. Relative training intensity and increases in strength in older women. Journal of Strength \& Conditioning Research, Colorado Springs, v. 9, n. 3, p. 188-191, aug. 1995.

INSTITUTO BRASILEIRO DE GEOGRAFIA E ESTATÍSTICA (IBGE). Disponível em: <unw.ibge.gov.br>. Acesso em: 22 out. 2009.

IZQUIERDO, M. et al. Maximal strength and power, endurance performance, and serum hormones in middle-aged and elderly men. Medicine and Science in Sports and Exercise, Hagerstown, v. 33, n. 9, p. 1577-1597, sep. 2001.

IZQUIERDO, M. et al. Once weekly combined resistance and cardiovascular training in healthy older men. Medicine and Science in Sports and Exercise, Hagerstown, v. 36, n. 3, p. 435-443, mar. 2004.

JANSSEN, I. et al. The healthcare costs of sarcopenia in the United States. Journal of the American Geriatrics Society, New York, v. 52, n. I, p. 80-85, jan. 2004.

KOSTKA, T. et al. Leg extensor power and dehydroepiandrosterone sulfate, insulin-like growth factor-I and testosterone in healthy active elderly people. European Journal Applied Physiology, Heidelberg, v. 82, n. I/2, p. 83-90, may 2000.

KOSTKA, T. Quadriceps maximal power and optimal shortening velocity in 335 men aged 23 88 years. European Journal Applied Physiology, Heidelberg, v. 95, n. 2/3, p. 140- I45, oct. 2005.

LIMA, R. M. et al. Fat-free mass, strength, and sarcopenia are related to bone mineral density in older women. Journal of Clinical Densitometry, v. I2, n. I, p. 35-4I, jan. 2009.

LINDLE, R. S. et al. Age and gender comparisons of muscle strength in 654 women and men aged 20-93yr. Journal of Applied Physiology, Bethesda, v. 83, p. I 58 I- I 587, nov. 1997.

MALKIA, E. et al. The physical activity of healthy and chronically ill adults in Finland at work, at leisure and during commuting. Scandinavian Journal of Medicine and Science in Sports, v. 4, n. I, p. 82-87, jan. 1994.

OLIVEIRA, R. J. et al. Association between sarcopenia-related phenotypes and aerobic capacity of older women. Journal of Sports Science and Medicine, v. 8, p. 337-343, sep. 2009.

ORDWAY, N. R. et al. Reliability of knee and ankle strength measures in an older adult population. Journal of Strength \& Conditioning Research, Colorado Springs, v. 20, n. I, p. 82-87, feb. 2006.

PORTER, M. M.; VANDERVOORT, A. A.; LEXELL, J. Aging of humans muscle: structure, function and adaptability. Scandinavian Journal of Medicine and Science in Sports, v. 5, n. 3, p. 129-142, jun. 1995.

RASO, V. et al. Exercícios com pesos para mulheres idosas. Revista Brasileira de Atividade Física e Saúde, Londrina, v. 2, n. 4, p. 17-26, 1997. 
RICE, C. L. et al. Arm and leg composition determined by computed tomography in young and eldery men. Clinical Physiology, v. 9, n. 3, p. 207-220, jun. 1989.

ROSENBERG, I. H. Epidemiologic and methodologic problems in determining nutritional status of older persons. American Journal of Clinical Nutrition, New York, v. 50, p. I 23 I - 1233 , nov. 1989.

ROTH, S. M.; FERRELL, R. E.; HURLEY, B. F. Strength training for prevention and treatment of sarcopenia. Journal of Nutrition Health and Aging, Paris, v. 4, n. 3, p. I43- I55, jan. 2000.

SCHUARTZ, R. S.; EVANS, W. J. Effects of exercise on body composition and functional capacity of the elderly The Journals of Gerontology Series A: Biological Sciences and Medical Sciences, v. 50A (special issue), p. 147-50, nov. 1995.

SILVA, T. A. A. et al. Sarcopenia Associada ao envelhecimento: Aspectos etiológicos e opções terapêuticas. Revista Brasileira de Reumatologia, São Paulo, v. 46, n.6, p. 39l-397, Nov./ dez. 2006.

STALBERG E. Macro EMG: a new recording technique. Journal of Neurology, Neurosurgey and Psychiatry, London, v. 43, p. 475-482, jun. 1980.

TRAPE, S., WILLIAMSON, D., GODARD, M. Maintenance of whole muscle strength and size following resistance training in older men. The Journals of Gerontology Series A: Biological Sciences and Medical Sciences, v. 57, p. BI38-BI 43, apr. 2002.

URSO, M. L.; CLARKSON, P. M.; PRICE, T. B. Immobilization effects in young and older adults. European Journal Applied Physiology, Heidelberg, v. 96, n. 5, p. 564-57I, mar. 2006.

Recebido: 12 jun. 2011 .

Aprovado: 4 mar. 2012

Endereço para correspondência:

Ricardo Moreno Lima

Universidade de Brasília (UnB), Campus Universitário Darcy Ribeiro, Faculdade de Educação Física, Asa Norte Brasilia - DF

CEP: 70910-970 